\title{
Guias carcinoma de celulas renales (SCU-2020)
}

\author{
Expertos temáticos: William Quiroga Matamoros ${ }^{1} \quad$ Jaime Iván Rangel $^{2}$ German Fabián Godoy Pérez ${ }^{3}$ \\ Fernando Fernández ${ }^{4}$ Grupo metodológico: Paula Andrea Camargo Zuluaga ${ }^{5}$ \\ Claudia Patricia Pérez Garavito ${ }^{6}$ Paola Andrea Orrego Rojas ${ }^{5}$
}

${ }^{1}$ Hospital Militar Central

2 Hospital Militar Central y Clínica Colsubsidio 127

3 Instituto Nacional de Cancerología

${ }^{4}$ Urólogo Fundación Puigvert, Universidad Autónoma Barcelona, Oncología Molecular Centro Nacional De Investigaciones Oncológicas De España , Servicio Urología Clínica Sebastian Belalcazar Cali

5 Universidad militar Nueva Granada

${ }^{6}$ Sanitas

Urol Colomb 2021;30:80-86.

La guía que se presenta actualmente es una adaptación de la guía de cáncer renal de la Asociación Europea de Urología en su versión más reciente 2020. Se eligió con la metodología ADOLOPMENT tras una evaluación con el instrumento AGREE II (-Table 1).

\section{Epidemiología}

El CCR representa el 2 a 3\% de todos los tipos de cáncer en el mundo. La incidencia para el 2018 de CCR en nuestro país es de 1793 nuevos casos por 100.000 habitantes, ocupando el puesto número 16 de diagnóstico y 17 de mortalidad, que corresponde al 1,5\% de causa de muerte por cáncer.

\section{Etiología}

Dentro de los factores etiológicos se incluyen el tabaquismo, la obesidad y la hipertensión. La diabetes y tener un familiar en primer grado con cáncer renal también se asocia a un incremento en el riesgo de CCR

\section{Patología}

Existen tres principales tipos: células claras (CCRcc), papilar (CCRp tipo II y II) y cromófobo (CCRc). En la actualidad el sistema de la OMS/ISUP han reemplazado el grado Fuhrman ${ }^{1}$. CCRcc es el subtipo más frecuente, se caracteriza por ser una lesión bien delimitadas con cápsula ausente. Se asocia a la pérdida del cromosoma 3p
Address for correspondence Paola Andrea Orrego Rojas, Universidad militar Nueva, Granada (e-mail: paolaorrego1206@gmail.com).

y mutaciones del gen de Von Hippel Lindau (VHL). Tiene el peor pronóstico 2,3

Las lesiones quísticas se clasifican de acuerdo con la clasificación de bosniak en su actualizacion 2019. Se basa principalmente en imágenes siendo la tomografía y la ultrasonografía con contraste lo ideal. La prevalencia de malignidad es de alrededor del $51 \%$ en bosniak III y $89 \%$ en bosniak IV.

\section{Evaluacion diagnóstica}

Más del $50 \%$ de los casos se diagnostican como masas sospechosas visualizadas en estudios radiológicos. ${ }^{4,5}$ La triada clásica de hematuria, dolor en flanco y masa palpable se correlaciona con histología agresiva y enfermedad avanzada ${ }^{6,7}$

La evaluación paraclínica incluye hemograma y panel metabólico completo. ${ }^{8}$ En el caso de masas centrales considere excluir carcinoma urotelial. Se debe estimar la función diferencial por gammagrafía cuando la función renal está comprometida o sea clínicamente relevante.

Se debe realizar Tomografía abdominal con contraste. Permite identificar la función del riñón contralateral, extensión del tumor primario, compromiso venoso, adenopatías locoregionales y el estado de otros órganos sólidos. La resonancia resulta útil en caso de quistes renales complejos y evaluación de compromiso venoso.
DOI https://doi.org/ $10.1055 / \mathrm{s}-0041-1726077$. ISSN 0120-789X. e ISSN 2027-0119. (c) 2021. Sociedad Colombiana de Urología. All rights reserved. This is an open access article published by Thieme under the terms of the Creative Commons Attribution-NonDerivative-NonCommercial-License, permitting copying and reproduction so long as the original work is given appropriate credit. Contents may not be used for commercial purposes, or adapted, remixed, transformed or built upon. (https://creativecommons.org/ licenses/by-nc-nd/4.0/)

Thieme Revinter Publicações Ltda., Rua do Matoso 170, Rio de Janeiro, RJ, CEP 20270-135, Brazil 
Table 1 Resumen de las recomendaciones

\begin{tabular}{|c|c|}
\hline Recomendación & Grado - NE \\
\hline $\begin{array}{l}\text { Incrementar la actividad física y eliminar el tabaquismo, así como la reducción de peso son medidas preventivas } \\
\text { para disminuir el riesgo de CCR. }\end{array}$ & Fuerte $-2 a$ \\
\hline Tratar los quistes Bosniak tipo III como CCR u ofrecer vigilancia activa & Débil - 2 \\
\hline Tratar los quistes Bosniak tipo IV de la misma manera que CCR & Fuerte -2 \\
\hline $\begin{array}{l}\text { Tratar los AMLs con embolización arterial selectiva o cirugía preservadora de nefronas en caso de: } \\
\text { - Tumores grandes (no hay un tamaño mínimo recomendado) } \\
\text { - Mujeres en edad fértil } \\
\text { - Pacientes con acceso difícil al sistema de salud para el seguimiento } \\
\text { - Dolor persistente o agudo, o episodios de sangrado recurrentes }\end{array}$ & Débil - 2 \\
\hline $\begin{array}{l}\text { Ofrecer vigilancia activa en pacientes con biopsia positiva para oncocitomas, como una alternativa aceptable a } \\
\text { cirugía o ablación }\end{array}$ & Débil - 3 \\
\hline $\begin{array}{l}\text { Emplee tomografía multifase con contraste de abdomen para el diagnóstico y estadificación de los tumores } \\
\text { renales }\end{array}$ & Fuerte - 2 \\
\hline $\begin{array}{l}\text { Emplee resonancia para evaluar el compromiso venoso, reducir la radiación y evitar la administración de } \\
\text { contraste endovenoso }\end{array}$ & Débil - 2 \\
\hline $\begin{array}{l}\text { Emplee modalidades no ionizantes, principalmente ultrasonografía con contraste (si dispone de ella), para } \\
\text { caracterizar las masas renales pequeñas y diferenciar masas inconclusas }\end{array}$ & Débil - 2 \\
\hline No realice de forma rutinaria gamagrafía ósea y/o PET para la estadificación del carcinoma de células renales & Débil - 3 \\
\hline Realice una biopsia del tumor antes de terapia ablativa o terapia sistémica sin patología previa & Fuerte -2 \\
\hline Realice biopsia percutánea en pacientes seleccionados que son considerados para vigilancia activa & Débil - 3 \\
\hline Emplee la técnica coaxial cuando vaya a realizar una biopsia & Fuerte -2 \\
\hline No realice biopsia renal a masas quísticas & Fuerte $-2 b$ \\
\hline Utilice técnica de Trucut en vez de aspiración con aguja fina para la caracterización histológica de masas sólidas & Fuerte -2 \\
\hline Emplee los sistemas de grado histológico para clasificar el tipo de CCR & Fuerte -2 \\
\hline $\begin{array}{l}\text { En enfermedad localizada, emplee sistemas pronósticos integrados o nomogramas para evaluar el riesgo de } \\
\text { recurrencia }\end{array}$ & Fuerte- 2 \\
\hline Emplee sistemas pronósticos en el escenario de enfermedad metastásica & Fuerte -2 \\
\hline Ofrecer vigilancia activa en pacientes frágiles y/o con múltiples comorbilidades con masas renales pequeñas & Débil - 3 \\
\hline Ofrecer cirugía como una terapia curativa en CCR localizado & Fuerte $-1 b$ \\
\hline Ofrecer nefrectomía parcial a pacientes con tumores T1 & Fuerte $-1 b$ \\
\hline $\begin{array}{l}\text { Ofrecer nefrectomía radical laparoscópica a pacientes con tumores T2 y masas localizadas no susceptibles de } \\
\text { nefrectomía parcial }\end{array}$ & Fuerte $-1 b$ \\
\hline $\begin{array}{l}\text { No realizar nefrectomía radical en pacientes con tumores T1 en quienes la nefrectomía parcial sea posible, } \\
\text { inclusive abierta }\end{array}$ & Fuerte $-2 b$ \\
\hline $\begin{array}{l}\text { No ofrezca cirugía mínimamente invasiva si esta puede comprometer los desenlaces oncológicos, funcionales } \\
\text { o perioperatorios }\end{array}$ & Fuerte - 2b \\
\hline $\begin{array}{l}\text { La nefrectomía parcial se puede realizar bien sea abierta, laparoscópica o asistida por robot, basado en la } \\
\text { experiencia y destrezas del cirujano }\end{array}$ & Fuerte $-2 b$ \\
\hline No realizar adrenalectomía ipsilateral si no hay evidencia clínica de invasión de la glándula & Fuerte -3 \\
\hline $\begin{array}{l}\text { Considerar linfadenectomía extendida en pacientes con características clínicas adversas incluyendo un } \\
\text { diámetro tumoral grande o características sarcomatoides }\end{array}$ & Débil - 3 \\
\hline $\begin{array}{l}\text { Ofrecer embolización en pacientes no aptos para manejo quirúrgico que se presenten con hematuria masiva o } \\
\text { dolor en flanco }\end{array}$ & Débil - 3 \\
\hline $\begin{array}{l}\text { Ofrecer vigilancia activa, ablación por radiofrecuencia o crio ablación en pacientes frágiles y/o comórbidos con } \\
\text { masas renales pequeñas }\end{array}$ & Débil - 3 \\
\hline $\begin{array}{l}\text { Informar a los pacientes en quienes se ofrecen terapias ablativas sobre el mayor riesgo de recurrencia local y/o } \\
\text { progresión tumoral }\end{array}$ & Débil -3 \\
\hline En pacientes sin metástasis, realice nefrectomía y trombectomía de la cava & Fuerte -3 \\
\hline $\begin{array}{l}\text { En pacientes con ganglios aumentados de tamaño clínicamente, realice linfadenectomía con propósitos de } \\
\text { estadificación o control local }\end{array}$ & Débil - 3 \\
\hline
\end{tabular}


Table 1 (Continued)

\begin{tabular}{|c|c|}
\hline No ofrecer terapia adyuvante con sorafenib, pazopanib o axitinib & Fuerte - 1b \\
\hline No ofrecer terapia adyuvante con sunitinib en pacientes con CCR células claras de alto riesgo & Débil - 1b \\
\hline $\begin{array}{l}\text { No ofrecer NC en pacientes con CCR metastásico con pobre riesgo MSKCC, a menos que la condición clínica lo } \\
\text { amerite }\end{array}$ & Fuerte $-1^{a}$ \\
\hline $\begin{array}{l}\text { Discutir la NC diferida en pacientes con riesgo intermedio MSKCC en tratamiento con VEGFR- ITK que puedan } \\
\text { obtener un beneficio a largo plazo y/o carga metastásica mínima residual }\end{array}$ & Débil -3 \\
\hline $\begin{array}{l}\text { Realice NC inmediata en pacientes con oligometástasis cuando el tratamiento local de las metástasis se puede } \\
\text { realizar de forma completa }\end{array}$ & Débil - 3 \\
\hline Realice la NC inmediata en pacientes con buen estado funcional que no requieran terapia sistémica & Débil -3 \\
\hline $\begin{array}{l}\text { Considerar terapia local para la enfermedad metastásica (metastasectomía) en pacientes con riesgo } \\
\text { favorable en los cuales la resección completa es técnicamente posible, para control de síntomas } \\
\text { locales }\end{array}$ & Débil - 3 \\
\hline $\begin{array}{l}\text { Ofrezca radioterapia estereotáctica para pacientes con enfermedad ósea relevante o metástasis cerebrales } \\
\text { para el control local y alivio sintomático }\end{array}$ & Débil -3 \\
\hline No ofrecer quimioterapia como tratamiento en pacientes con CCR metastásica & Fuerte - 1b \\
\hline $\begin{array}{l}\text { Ofrecer pembrolizumab + axitinib para el tratamiento de pacientes con CCRcc metastásico, cualquier IMDC sin } \\
\text { tratamiento previo }\end{array}$ & Fuerte - 1b \\
\hline $\begin{array}{l}\text { Ofrecer Nivolumab + Ipilimumab en pacientes con CCR cc metastásico IMDC intermedio o pobre sin } \\
\text { tratamiento previo }\end{array}$ & Fuerte $-1^{\mathrm{a}}$ \\
\hline $\begin{array}{l}\text { Ofrecer nivolumab como monoterapia en pacientes que no toleran las } 4 \text { dosis de ipilimumab por toxicidad, } \\
\text { mientras sea seguro y posible }\end{array}$ & Débil \\
\hline Ofrecer Nivolumab después de una o dos terapias con VEGF en CCR metastásico & Fuerte - 1b \\
\hline $\begin{array}{l}\text { Ofrecer sunitinib o pazopanib en pacientes sin tratamiento previo, con cualquier IMDC que no puedan tolerar o } \\
\text { recibir inhibidores de punto de chequeo }\end{array}$ & Fuerte $-1 b$ \\
\hline $\begin{array}{l}\text { Ofrecer nivolumab o cabozantinib para pacientes sin tratamiento previo con inhibidores del punto de chequeo } \\
\text { y refractarios al tratamiento con anti- VEGFR }\end{array}$ & Fuerte - 2b \\
\hline $\begin{array}{l}\text { La secuenciación de agentes no usados en segunda línea (nivolumab o cabozantinib) se recomiendan para } \\
\text { tercera línea }\end{array}$ & Débil 3 \\
\hline $\begin{array}{l}\text { Ofrecer inhibidores de la tirosina quinasa- VEGFR en segunda línea a pacientes refractarios a nivolumab + } \\
\text { ipilimumab o axitinib + pembrolizumab }\end{array}$ & Débil 2b \\
\hline Ofrecer cabozantinib después de terapia con anti VEGFR en células claras & Fuerte - 1b \\
\hline Secuenciar terapia sistémica en paciente con CCR metastásico & Fuerte -3 \\
\hline $\begin{array}{l}\text { Ofrecer resección quirúrgica de la enfermedad localmente recurrente cuando sea posible una resección } \\
\text { completa y en ausencia de comorbilidades }\end{array}$ & Débil 3 \\
\hline Basar el seguimiento en el riesgo de recurrencia & Fuerte 4 \\
\hline $\begin{array}{l}\text { Intensificar el seguimiento en pacientes después de cirugía preservadora de nefronas }>7 \mathrm{~cm} \text { o en pacientes con } \\
\text { márgenes positivos }\end{array}$ & Débil 3 \\
\hline $\begin{array}{l}\text { Basar el riesgo en clasificaciones preexistentes como el sistema integrado de estadificación de la Universidad } \\
\text { de California Los Ángeles o el puntaje de SSIGN }\end{array}$ & Fuerte 2 \\
\hline
\end{tabular}

La tomografía de tórax es precisa para la estatificación ${ }^{9}$ y solo en casos de síntomas se puede realizar gammagrafía ósea, tomografía cerebral o resonancia. ${ }^{10-12}$

\section{Biopsia renal}

Se puede considerar en pacientes candidatos a vigilancia activa, diagnóstico histológico previo a terapias ablativas y en el escenario de enfermedad metastásica ${ }^{13-18}$

La sensibilidad y especificidad con técnica trucut es de $99,1 \%$ y $99,7 \%$ respectivamente $^{19}$ Hasta el $22,6 \%$ de las biopsias no van a ser diagnósticas ${ }^{13,16,20-22}$ ). La tasa de concordancia es del $90,3 \%{ }^{19}$

\section{Estadificacion y Pronóstico}

El sistema de clasificación recomendado es el $\mathrm{TNM}^{23}$

Existen diferentes factores pronósticos lo cuales se deben clasificar en anatómicos, histológicos, clínicos y moleculares.

Existen nomogramas y sistemas pronósticos post operatorios que combinan factores pronósticos independientes. Los 
disponibles para el CCR localizado son UISS, SSIGN y Karakiewicz. En el escenario de enfermedad metastásica están el sistema pronóstico de Memorial Sloan Kettering Cancer Center (MSKCC) (121)y el modelo de Heng (IMDC) (122)

\section{Tratamiento}

\section{Enfermedad localizada}

\section{Vigilancia}

La vigilancia activa se define como la monitorización del tamaño tumoral con imágenes seriadas, y una intervención retrasada reservada para tumores que demuestran progresión clínica durante el seguimiento (126)

La vigilancia activa no fue predictiva de supervivencia global o mortalidad cáncer específica en modelos de regresión con seguimiento relativamente corto (127).

\section{Manejo quirúrgico}

y considerando la seguridad oncológica, en tumores T1b el tratamiento de elección es la $\mathrm{CP}$, considerando que preserva la función renal y limita a largo plazo las alteraciones cardiovasculares potenciales.

La realización de la adrenalectomía no impactó en la supervivencia global a 10 años. Solo se debe considerar en caso de compromiso intraoperatorio o radiológico(163).

La indicación para realizarla es controversial (164) ya que no hay beneficios claros en supervivencia. En caso de que se considere la realización de la linfadenectomía debería incluir grandes vasos ipsilaterales y la región inter aortocava desde la crura diafragmática hasta la arteria iliaca común. Se recomienda que al menos se extraigan 15 ganglios(168-171).

\section{Enfermedad localmente avanzada}

Se debe considerar por tanto que independiente de la extensión del trombo, en pacientes sin enfermedad metastásica y un buen estado funcional se les debe practicar manejo quirúrgico. Su selección depende del nivel del trombo y el grado de oclusión de la vena cava inferior (186-188).

En presencia de enfermedad ganglionar clínicamente positiva la linfadenectomía siempre está justificada (189)

\section{Terapia adyuvante}

Actualmente no hay evidencia derivada que demuestren que la terapia adyuvante ofrezca un beneficio de supervivencia después de la cirugía.

\section{Enfermedad avanzada/metastásica}

\section{Nefrectomía citoreductora}

La resección tumoral es curativa solo si todas las siembras tumorales se resecan. Esto incluye pacientes con tumor primario y enfermedad oligometastásica resecable. Para la mayoría de los pacientes con enfermedad metastásica, la NC es paliativa y el tratamiento sistémico es necesario.

\section{Terapia sistémica}

Bloqueo de puntos de chequeo

Inhibición de los puntos de chequeo con anticuerpos monoclonales y el bloqueo inhibitorio de los receptores PD-1 de las células T o del antígeno citotóxico asociado a los linfocitos T (CTLA-4) tiene como objetivo restaurar la inmunidad mediada por las células T dirigida al tumor (206)

Los datos aleatorizados apoyan el uso del Nivolumab en la enfermedad refractaria a VEGF. La combinación de dos inhibidores (terapia combinada) Ipilimumab + nivolumab mostró una supervivencia superior en pacientes de riesgo intermedio y bajo, mientras que la combinación de pembrolizumab y axitinib mostró ventaja de supervivencia para pacientes de todos los grupos de riesgo. - Figura 1.

\section{Histología no células claras}

La histología más común no células claras es el subtipo papilar. Basado en una revisión sistemática que incluyó el

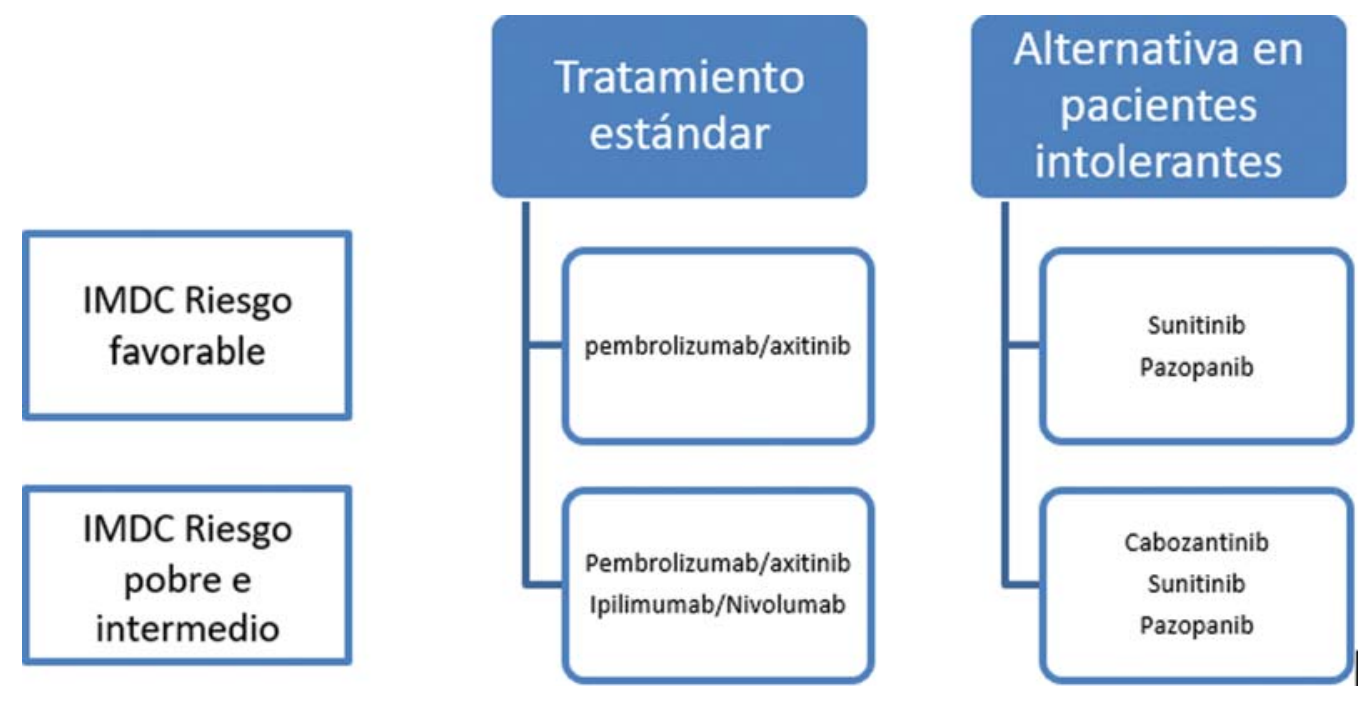

Fig. 1 Estrategia terapéutica. 
Tabla 2 Seguimiento propuesto

\begin{tabular}{|c|c|c|c|c|c|}
\hline \multirow[t]{2}{*}{ Perfil de riesgo } & \multicolumn{5}{|l|}{ Seguimiento } \\
\hline & $6 \mathrm{~m}$ & 1 año & 2 años & 3 años & $>3$ años \\
\hline Bajo & US & Tomografía & US & Tomografía & Tomografía cada 2 años \\
\hline Intermedio/Alto & Tomografía & Tomografía & Tomografía & Tomografía & Tomografía cada 2 años \\
\hline
\end{tabular}

estudio ESPN, RECORD-3 y ASPEN, el sunitinib y el everolimus son una opción en esta población. (194,239,240)

\section{Recurrencia}

La enfermedad localmente recurrente puede afectar el riñón en caso de la NP o terapia focal, o por fuera del riñón seguido de NR. El procedimiento de salvamento más efectivo alternativo es la resección quirúrgica. Los parámetros adversos son un tiempo de aparición corto <3-12m (246), diferenciación sarcomatoide de la recurrencia y una resección quirúrgica incompleta (244)

\section{Seguimiento}

El seguimiento busca monitorizar o identificar: complicaciones postoperatorias, función renal, recurrencia local o contralateral y desarrollo de metástasis.

Se propone un modelo individualizado basado en el riesgo, que incorporan la edad, estadio patológico, localización de recurrencia y comorbilidades para calcular cuándo el riesgo de muerte por otra causa excede el riesgo de recurrencia del CCR (247) (-Table 2).

\section{Referencias}

1 Moch H, Cubilla AL, Humphrey PA, Reuter VE, Ulbright TM. The 2016 WHO Classification of Tumours of the Urinary System and Male Genital Organs-Part A: Renal, Penile, and Testicular Tumours. Eur Urol 2016;70(01):93-105http://www.ncbi.nlm. nih.gov/pubmed/26935559 [Internet]

2 Brugarolas J. Molecular genetics of clear-cell renal cell carcinoma. J Clin Oncol 2014;32(18):1968-1976http://www.ncbi.nlm. nih.gov/pubmed/24821879 [Internet]

3 Capitanio U, Cloutier V, Zini L, et al. A critical assessment of the prognostic value of clear cell, papillary and chromophobe histological subtypes in renal cell carcinoma: a populationbased study. BJU Int 2009;103(11):1496-1500http://www.ncbi. nlm.nih.gov/pubmed/19076149 [Internet]

4 Novara G, La Falce S, Kungulli A, Gandaglia G, Ficarra V, Mottrie A. Robot-assisted partial nephrectomy. Int J Surg 2016;36(Pt C):554-559. Doi: 10.1016/j.ijsu.2016.05.073 [Internet]

5 Jayson M, Sanders H. Increased incidence of serendipitously discovered renal cell carcinoma. Urology 1998;51(02): 203-205http://www.ncbi.nlm.nih.gov/pubmed/9495698 [Internet]

6 Patard J-J, Leray E, Rodriguez A, Rioux-Leclercq N, Guillé F, Lobel B. Correlation between symptom graduation, tumor characteristics and survival in renal cell carcinoma. Eur Urol 2003;44(02): 226-232http://www.ncbi.nlm.nih.gov/pubmed/12875943 [Internet]

7 Lee CT, Katz J, Fearn PA, Russo P. Mode of presentation of renal cell carcinoma provides prognostic information. Urologic oncology
[Internet]7(04):135-140. Available from: http://www.ncbi.nlm. nih.gov/pubmed/12474528

8 Magera JS Jr, Leibovich BC, Lohse CM, et al. Association of abnormal preoperative laboratory values with survival after radical nephrectomy for clinically confined clear cell renal cell carcinoma. Urology 2008;71(02):278-282http://www.ncbi.nlm. nih.gov/pubmed/18308103 [Internet]

9 Heidenreich A, Ravery VEuropean Society of Oncological Urology. Preoperative imaging in renal cell cancer. World J Urol 2004;22 (05):307-315http://www.ncbi.nlm.nih.gov/pubmed/15290202 [Internet]

10 Marshall ME, Pearson T, Simpson W, Butler K, McRoberts W. Low incidence of asymptomatic brain metastases in patients with renal cell carcinoma. Urology 1990;36(04):300-302http:// www.ncbi.nlm.nih.gov/pubmed/2219605 [Internet]

11 Henriksson C, Haraldsson G, Aldenborg F, Lindberg S, Pettersson S. Skeletal metastases in 102 patients evaluated before surgery for renal cell carcinoma. Scand J Urol Nephrol 1992;26(04): 363-366http://www.ncbi.nlm.nih.gov/pubmed/1292074 [Internet]

12 Seaman E, Goluboff ET, Ross S, Sawczuk IS. Association of radionuclide bone scan and serum alkaline phosphatase in patients with metastatic renal cell carcinoma. Urology 1996;48 (05):692-695http://www.ncbi.nlm.nih.gov/pubmed/8911510 [Internet]

13 Shannon BA, Cohen RJ, de Bruto H, Davies RJ. The value of preoperative needle core biopsy for diagnosing benign lesions among small, incidentally detected renal masses. J Urol 2008;180 (04):1257-1261, discussion 1261http://www.ncbi.nlm. nih.gov/pubmed/18707712 [Internet]

14 Maturen KE, Nghiem HV, Caoili EM, Higgins EG, Wolf JS Jr, Wood DP Jr. Renal mass core biopsy: accuracy and impact on clinical management. AJR Am J Roentgenol 2007;188(02):563-570http:// www.ncbi.nlm.nih.gov/pubmed/17242269 [Internet]

15 Veltri A, Garetto I, Tosetti I, et al. Diagnostic accuracy and clinical impact of imaging-guided needle biopsy of renal masses. Retrospective analysis on 150 cases. Eur Radiol 2011;21(02): 393-401http://www.ncbi.nlm.nih.gov/pubmed/20809129 [Internet]

16 Abel EJ, Culp SH, Matin SF, et al. Percutaneous biopsy of primary tumor in metastatic renal cell carcinoma to predict high risk pathological features: comparison with nephrectomy assessment. J Urol 2010;184(05):1877-1881http://www.ncbi. nlm.nih.gov/pubmed/20850148 [Internet]

17 Volpe A, Mattar K, Finelli A, et al. Contemporary results of percutaneous biopsy of 100 small renal masses: a single center experience. J Urol 2008;180(06):2333-2337http://www.ncbi. nlm.nih.gov/pubmed/18930274 [Internet]

18 Richard PO, Jewett MAS, Bhatt JR, et al. Renal Tumor Biopsy for Small Renal Masses: A Single-center 13-year Experience. Eur Urol 2015;68(06):1007-1013http://www.ncbi.nlm.nih.gov/pubmed/ 25900781 [Internet]

19 Marconi L, Dabestani S, Lam TB, et al. Systematic Review and Meta-analysis of Diagnostic Accuracy of Percutaneous Renal Tumour Biopsy. Eur Urol 2016;69(04):660-673http://www. ncbi.nlm.nih.gov/pubmed/26323946 [Internet]

20 Leveridge MJ, Finelli A, Kachura JR, et al. Outcomes of small renal mass needle core biopsy, nondiagnostic percutaneous biopsy, and 
the role of repeat biopsy. Eur Urol 2011;60(03):578-584http:// www.ncbi.nlm.nih.gov/pubmed/21704449 [Internet]

21 Breda A, Treat EG, Haft-Candell L, et al. Comparison of accuracy of 14-, 18- and 20-G needles in ex-vivo renal mass biopsy: a prospective, blinded study. BJU Int 2010;105(07):940-945http:// www.ncbi.nlm.nih.gov/pubmed/19888984 [Internet]

22 Motzer RJ, Barrios CH, Kim TM, et al. Phase II randomized trial comparing sequential first-line everolimus and second-line sunitinib versus first-line sunitinib and second-line everolimus in patients with metastatic renal cell carcinoma. J Clin Oncol 2014;32(25):2765-2772http://www.ncbi.nlm.nih.gov/pubmed/ 25049330 [Internet]

23 Sobin LH, G.M WC. TNM classification of malignant tumors. In: UIUA Cancer [Internet] 2009. Available from: https://www. wiley.com/en-us/TNM+Classification+of+Malignant+Tumours\% 2C+7th+Edition-p-9781444358964 